\title{
EFEITOS AGUDOS DO TREINAMENTO AERÓBIO SOBRE O DESEMPENHO DA FORÇA MUSCULAR
}

\author{
Hugo Tourinho Filho \\ Universidade de São Paulo, Ribeirão Preto, São Paulo, Brasil \\ Enrico Fuini Puggina \\ Universidade de São Paulo, Ribeirão Preto, São Paulo, Brasil \\ Lucas Lima Marini \\ Universidade de Passo Fundo, Passo Fundo, Rio Grande do Sul, Brasil \\ Dalmo Roberto Lopes Machado \\ Universidade de São Paulo, Ribeirão Preto, São Paulo, Brasil \\ Valdir José Barbanti \\ Universidade de São Paulo, Ribeirão Preto, São Paulo, Brasil \\ Gilnei Lopes Pimentel \\ Universidade de Passo Fundo, Passo Fundo, Rio Grande do Sul, Brasil
}

\begin{abstract}
Resumo
Investigou-se as respostas agudas induzidas por uma série de treinamento aeróbio sobre o desempenho da força muscular. Para tal, atletas adultos realizaram uma série de trabalho aeróbio e, em seguida, executaram testes para inferir força explosiva e pico de torque de membros inferiores nas seguintes situações: em repouso, imediatamente após o trabalho aeróbio e depois de oito e 24 horas da realização do trabalho aeróbio. Com base nos resultados obtidos, foi possível verificar que o trabalho aeróbio realizado durante 20 minutos, a uma intensidade de $90 \%$ do limiar lático, não prejudicou o desempenho da força muscular nos diferentes momentos de recuperação, ou seja, nessa intensidade e duração de corrida, não se verificou o fenômeno da interferência para as variáveis analisadas.

Palavras-chave: Força Muscular. Educação Física e Treinamento. Desempenho Atlético.
\end{abstract}

\section{Introdução}

tletas de diferentes esportes realizam treinamento de força e re-
sistência aeróbia dentro de seus programas de preparação física
num esforço para alcançar adaptações específicas a ambas as formas
de treinamento. Entretanto, algumas pesquisas têm mostrado que o
desenvolvimento da força muscular pode ser prejudicado quando o
treinamento dessa capacidade motora é combinado com o treinamento 
de resistência aeróbia (DUDLEY; DJAMIL, 1985; HICKSON, 1980; KRAEMER et. al., 1995). Uma possível explicação para o suposto prejuízo no desenvolvimento da força muscular treinada juntamente com a resistência aeróbia, reside no fato de que programas de treinamento de força e resistência aeróbia são utilizados para induzir respostas adaptativas completamente diferentes, daí o termo "treinamentos concorrentes". Nesse sentido, a natureza das respostas adaptativas ao treinamento é específica ao estímulo do treinamento (HICKSON, 1980), e a possibilidade de incompatibilidade entre as adaptações específicas provocadas por uma ou outra forma de treinamento atualmente vem sendo denominada de "fenômeno de interferência" (BELL et. al., 1997; DOCHERTY; SPORER, 2000; TOURINHO, 2007, 2010).

Apesar das dificuldades em se comparar os resultados dos estudos sobre treinamentos concorrentes em função das diferenças observadas entre os designs dessas pesquisas, a literatura tem sinalizado para a existência de duas hipóteses que tentam explicar os possíveis mecanismos responsáveis pela diminuição do rendimento de força e resistência aeróbia ao serem treinados simultaneamente (fenômeno da interferência): hipótese crônica e hipótese aguda (LEVERITT et. al., 1999).

Na hipótese crônica é defendida a ideia de que a musculatura esquelética não pode adaptar-se metabólica e morfologicamente para ambos os treinamentos, simultaneamente. Isso se deve ao fato de que muitas adaptações em nível muscular observadas em resposta ao treinamento de força são diferentes daquelas observadas após o treinamento de resistência aeróbia (aumento da atividade enzimática glicolítica, hipertrofia de fibras brancas x aumento da densidade mitocondrial, aumento da atividade enzimática oxidativa, etc.). Já, com relação à hipótese aguda, defende-se a ideia de que a instalação de uma fadiga residual periférica provocada pelo treinamento aeróbio prejudicaria a capacidade de desenvolver tensão durante o treinamento de força. Fatores relacionados à fadiga, tais como depleção de substratos energéticos como ATP, fosfocreatina e glicogênio na musculatura treinada, além de acúmulo de metabólitos (fosfato inorgânico lactato e amônia) têm sido implicados como possível mecanismo associado com a hipótese aguda (LEVERITT et al., 1999). Atualmente, tanto a hipótese crônica como a hipótese aguda têm sido propostas para explicar o fenômeno da inibição do desempenho (fenômeno da 
interferência) verificado por alguns estudos, quando são combinadas força e resistência aeróbia no mesmo modelo de treinamento (HICKSON, 1980; DUDLEY; DJAMIL, 1985; KRAEMER et al., 1995; NELSON et al., 1990; DOLEZAL; POTTEIGER, 1998; GRAVELLE; BLESSING, 2000).

Dessa maneira, considerando o que foi exposto, o presente estudo teve como objetivo investigar o comportamento da força muscular avaliada imediatamente após, oito e 24 horas depois da realização de uma série de trabalho aeróbio.

\section{Material e métodos}

Amostra

A amostra foi selecionada de forma não-aleatória, sendo composta por seis atletas voluntários de uma equipe adulta de judô $(23+/-3,4$ anos), com um tempo médio de prática de $12 \pm 3$ anos. O tamanho da amostra foi determinado por conveniência, mediante a disponibilidade e aceitação dos sujeitos em participar do estudo.

Coleta de dados

O presente estudo atendeu as normas da Resolução 196/96 do Conselho Nacional da Saúde de 10/10/96 para a realização de pesquisa em seres humanos e foi submetido e aprovado pelo Comitê Local de Ética em Pesquisa.

Após assinarem o termo de consentimento, os indivíduos compareceram ao laboratório em seis momentos distintos para a realização da bateria de testes proposta pelo presente estudo (Figura 1).

No primeiro dia, os avaliados compareceram ao laboratório para a determinação da força explosiva avaliada por meio da realização de saltos verticais (SJ e CMJ) e para a realização de medidas antropométricas. A obtenção do pico de torque dos membros inferiores foi realizada no segundo dia de visita ao laboratório. No terceiro dia, foi realizado um teste progressivo em esteira rolante para a determinação do limiar lático, que possibilitou dosar, de forma individualizada, a intensidade da série de trabalho aeróbio a ser realizada na pesquisa.

Em um segundo momento do estudo (quarta, quinta e sexta visitas), os indivíduos realizaram a série de trabalho aeróbio e os testes de 
força tanto no ergojump como no isocinético Biodex, nas situações: imediatamente após, ou então, oito e $24 \mathrm{~h}$ após a realização do trabalho aeróbio.

A escolha dos intervalos de oito e 24 horas foi determinada baseando-se no tempo de intervalo que, geralmente, os atletas têm entre uma sessão e outra de treinamento de resistência aeróbia e força. No caso das oito horas, seria o intervalo entre o trabalho aeróbio realizado pela parte da manhã e o treinamento de força realizado no período vespertino. Já, para as 24 horas, esse intervalo estaria simulando um treinamento de força realizado no dia seguinte ao treinamento aeróbio.

A seqüência de trabalho aeróbio e o tempo de recuperação para a realização dos testes de força foram sorteados para cada atleta, e um tempo de 48 horas foi dado entre uma seqüência e outra de trabalho aeróbio e testes de força, a fim de se obter um tempo mínimo de recuperação para os atletas.

Entre os intervalos de recuperação, os indivíduos foram orientados a evitar qualquer outro tipo de atividade física.

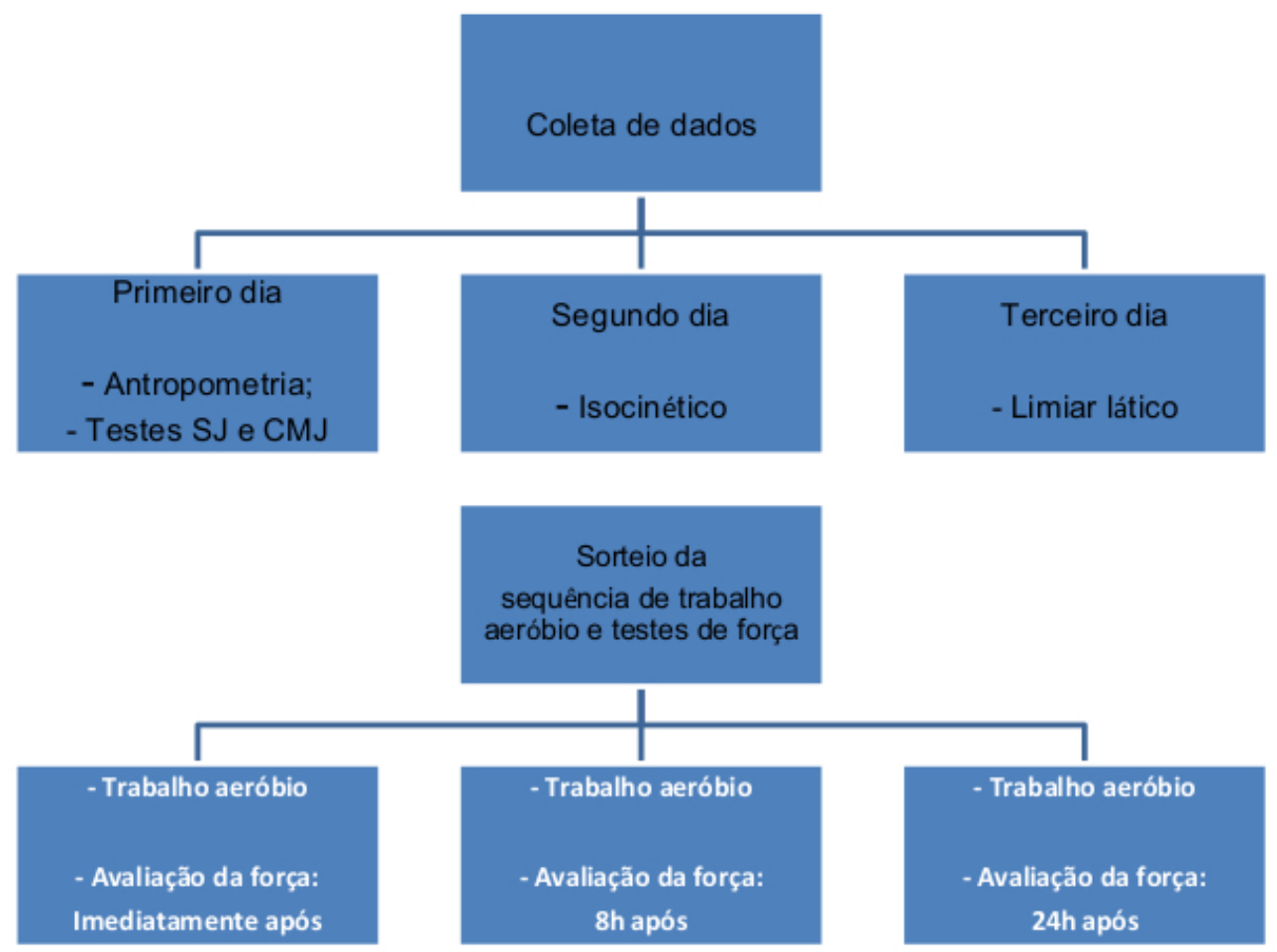

Figura 1: Organograma dos procedimentos realizados na coleta de dados 
Variáveis de estudo

Determinação do limiar lático

Para a determinação da intensidade do trabalho aeróbio foi utilizado um teste escalonado em esteira rolante 10200 AT (IMBRAMED ${ }^{\circledR}$, Brasil), determinando-se o limiar lático, medido através do lactato a ponto fixo em $3,5 \mathrm{mmol} / \mathrm{L}$ de acordo com o protocolo sugerido por Heck et al. (1985). Ao final de cada estágio, foi anotada a freqüência cardíaca com um monitor de freqüência cardíaca tipo Polar modelo Sport Tester TM GBR165020 (Polar®, Finlândia).

O sangue, para a análise do lactato foi coletado do lóbulo da ore1ha. Com o intuito de promover vasodilatação para facilitar a obtenção do sangue arterializado, foi utilizada a pomada de nome comercial Finalgon Cream (Boehringer Ingelheim PTY Limited ${ }^{\circledR}$, Austrália). O sangue é automaticamente decantado sobre uma fita de medição do aparelho Accu-Sports, que apresenta a característica de um minifotômetro (Gambke et al., 1994), mostrando-se um instrumento válido e fidedigno para a faixa de concentração de lactato sangüíneo em que ocorre o limiar anaeróbio (FELL et al., 1998).

Prescrição da série de trabalho aeróbio

A série de trabalho aeróbio foi prescrita com base na determinação do limiar lático, que permite dosar a intensidade do trabalho aeróbio respeitando os limites de cada indivíduo. O trabalho aeróbio foi prescrito em uma intensidade realizada a $90 \%$ da velocidade em que ocorreu o limiar lático (OLIVERIA; GAGLIARDI; KISS, 1994). Essa velocidade foi escolhida por representar uma intensidade que pode ser utilizada na preparação física de atletas em início de temporada. Os atletas realizaram a corrida com duração de 20 minutos em uma esteira rolante.

Teste de salto vertical

A fim de se inferir o comportamento da força explosiva de membros inferiores, foram utilizados testes de saltos verticais (altura de elevação do centro de gravidade) com o auxílio do equipamento ergojump (Jump Test Pro ${ }^{\circledR}$, Brasil) - tapete composto de circuitos eletrô- 
nicos que medem o tempo que o indivíduo fica sem o contato com o equipamento durante a execução do salto (BOSCO et al., 1995).

O equipamento ergojump possui um software (Jump Test System 1.2) que utiliza as equações propostas por Bosco et al. (1995) para calcular a altura de elevação do centro de gravidade em centímetros (d $=1 / 8 \mathrm{gt} 2$ ), com precisão de uma casa decimal, onde:

$\mathrm{d}=$ elevação máxima do centro de gravidade;

$\mathrm{g}=$ gravidade;

$\mathrm{t}=$ tempo de vôo.

a) Salto partindo da posição de semi-agachamento - SQUAT JUMP (SJ)

Os indivíduos posicionaram-se em pé, em cima do tapete do ergojump, pés paralelos, mãos nos quadris, com os joelhos flexionados a 90 (medido com o auxílio de um goniômetro manual Sanny®, Brasil, fixado com seu centro de rotação na linha intercondilar lateral do joelho direito). Ao avaliado foi permitida a execução de nova flexão do joelho, devendo, dessa maneira, partir da posição predeterminada para o salto vertical. Para efeito dos cálculos estatísticos, foi considerado o melhor salto de três tentativas que foram realizadas com a técnica SJ (SZMUCHROWSKI; VIDIGAL, 1999).

b) Salto com contramovimento - COUNTERMOVEMENT JUMP (CMJ)

Os indivíduos posicionaram-se em pé sobre o tapete do equipamento ergojump, com os pés paralelos e as mãos nos quadris. Nesse modelo de salto, foram permitidas a flexão e a extensão dos membros inferiores livremente. A transição da primeira fase (descendente) para a fase seguinte (ascendente) deve ocorrer por intermédio de um movimento contínuo, no qual as articulações são estendidas, devendo essas extensões ser feitas o mais rapidamente possível.

Para efeito dos cálculos estatísticos, foi considerado o melhor salto de três tentativas que foram realizadas com a técnica CMJ (SZMUCHROWSKI; VIDIGAL, 1999).

Avaliação do pico de torque (N) - biodex multijoint system 3 pro

Para a avaliação do pico de torque (PT) foi utilizado o equipamento Biodex Multijoint System 3 Pro (Biodex ${ }^{\circledR}$, EUA), no seu modo 
Isocinético. $\mathrm{O}$ atleta foi posicionado na cadeira e recebeu informações sobre os procedimentos que seriam realizados. O dinamômetro foi deslocado ao longo do plano horizontal, posicionando-se na face externa do membro inferior não dominante, padronizado para ser o primeiro a ser testado. Conectou-se o acessório do joelho ao dinamômetro e alinhou-se o eixo de rotação do joelho do atleta com o eixo do dinamômetro. Ajustou-se a altura do assento na direção do dinamômetro, ou no sentido contrário, para obter ajuste fino. Estabilizou-se o atleta com um par de cintos de ombro, cinto pélvico e um cinto para a coxa a ser testada. Finalizaram-se os preparativos com a instalação das paradas de amplitude de movimento. A efetivação da ação deu-se por meio de uma série de dez movimentos de extensão e flexão do joelho, de maneira concêntrica/concêntrica, na velocidade de $60^{\circ} / \mathrm{s}$. Logo após, foi permitido um período de repouso de 30 segundos antes da segunda série de dez repetições do mesmo movimento, porém com uma velocidade de $180 \%$ s. Um novo intervalo de 30 segundos foi concedido, e a avaliação deste membro foi finalizada com dez movimentos de flexo-extensão a uma velocidade de $300 \%$ s. Concluído o protocolo de avaliação do membro inferior não dominante, imediatamente após, o dinamômetro foi deslocado para o lado oposto e o procedimento foi repetido com o membro inferior dominante. Ao término do teste, foram coletados os valores do pico de torque obtidos durante as dez repetições dos músculos envolvidos (flexores e extensores do joelho) em cada uma das séries de exercícios nas velocidades referentes a $60 \%$ s, $180^{\circ} / \mathrm{s}$ e $300 \%$ s bilateralmente. O protocolo de avaliação de força isocinética foi adaptado do estudo de Leveritt e Abernethy (1999).

Tratamento estatístico

A fim de atender aos objetivos propostos para o estudo, as informações coletadas foram tratadas estatisticamente mediante o uso do pacote computadorizado Statistic for Windows, adotando-se o seguinte procedimento: para verificar a normalidade dos dados foi realizado o Teste Shapiro-Wilk; para comparar o comportamento da força muscular nos diferentes momentos de avaliação, foi utilizada uma análise de variância para medidas repetidas (NETER et al., 1996). O teste de comparações múltiplas Post-Hoc de Tukey foi empregado na identificação das diferenças específicas quando os valores de "F" encontrados 
mostraram-se superiores ao critério de significância estatística estabelecido, predeterminado aos níveis de 0,05 .

\section{Resultados}

A fim de se descrever as características da amostra estudada, os atletas realizaram uma bateria de medidas antropométricas para se determinar a estatura, massa corporal, percentual de gordura e massa magra e teste para determinação do limiar lático (Tabela 1).

Tabela 1: Valores médios de estatura, massa corporal, percentual de gordura, massa magra, velocidade de corrida referente ao limiar anaeróbio (VCL), e velocidade de corrida a $90 \%$ do limiar anaeróbio $\left(\mathrm{VCL}_{90}\right)$.

\begin{tabular}{llcc}
\hline \multicolumn{1}{c}{ Variáveis } & n & Média & Desvio - padrão \\
\hline Estatura $(\mathrm{cm})$ & 6 & 175,05 & 8,12 \\
Massa corporal $(\mathrm{kg})$ & 6 & 78,31 & 12,70 \\
\% de gordura & 6 & 14,65 & 3,11 \\
Massa magra $(\mathrm{kg})$ & 6 & 66,57 & 8,56 \\
$\operatorname{VCL}(\mathrm{km} / \mathrm{h})$ & 6 & 8,98 & 1,62 \\
$\mathrm{VCL}_{90}(\mathrm{~km} / \mathrm{h})$ & 6 & 8,08 & 1,46 \\
\hline
\end{tabular}

Nas Tabelas 2, 3 e 4 encontram-se os valores de força explosiva (SJ) e força explosiva elástica (CMJ), avaliadas nos diferentes momentos de recuperação após a realização do trabalho aeróbio executado durante 20 minutos a uma intensidade de $90 \%$ da velocidade referente ao limiar lático.

Por intermédio da análise estatística, foi possível verificar diferenças estatisticamente significantes entre os valores obtidos para a força explosiva (SJ) nas situações de repouso (Base) em relação aos valores obtidos imediatamente após a realização do trabalho aeróbio (Ime) e 8 horas após a execução da corrida, e entre as situações Ime e 8 horas com a avaliação realizada após 24 horas da corrida. Nestes testes, os valores de força explosiva obtidos sem a corrida precedendo o teste (Base) e os resultados obtidos após 24 horas, apresentaram-se mais baixos que os obtidos imediatamente após a corrida e 8 horas após a realização do trabalho aeróbio.

Com relação ao teste de impulsão vertical que buscou inferir a força explosiva elástica (CMJ), observou-se diferenças significativas apenas entre os resultados obtidos imediatamente após a corrida (Ime) 
e os resultados apresentados pelos atletas após 24 horas de intervalo da corrida. Os valores de Ime mostraram-se superiores aos obtidos com o intervalo de 24 horas de recuperação.

Tabela 2: Valores médios referentes aos testes de impulsão vertical (SJ e CMJ), realizados em repouso (Base), imediatamente após a realização de uma série de trabalho aeróbio (Ime) e após 8 e 24 horas da execução da corrida.

\begin{tabular}{lccccc}
\hline Teste de saltos & Base & Ime & $\mathbf{8 h}$ & $\mathbf{2 4 h}$ & "p” \\
\hline SJ $(\mathrm{cm})$ & $35,0 \pm 4,5$ & $37,8 \pm 4,74$ & $37,5 \pm 5,1$ & $34,5 \pm 4,2$ & $0,001^{*}$ \\
CMJ (cm) & $38,2 \pm 5,2$ & $40,0 \pm 5,2$ & $39,1 \pm 5,8$ & $36,4 \pm 3,9$ & $0,01^{*}$ \\
\hline
\end{tabular}

$* \mathrm{p} \leq 0,05$

Tabela 3: Resultados do Post Hoc de Tuckey para o teste de impulsão vertical (SJ), realizado pelos atletas.

\begin{tabular}{lcccc}
\hline \multicolumn{1}{c}{ Variáveis } & Base (35,0 $\mathbf{c m})$ & Ime $\mathbf{( 3 7 , 7 8 ~} \mathbf{~ c m})$ & $\mathbf{8 h} \mathbf{( 3 7 , 5 2} \mathbf{~ c m})$ & $\mathbf{2 4 h} \mathbf{( 3 4 , 5} \mathbf{~ c m})$ \\
\hline Base & ---- & $0,01^{*}$ & $0,03^{*}$ & 0,92 \\
Ime & --- & --- & 0,99 & $0,005^{*}$ \\
8hrs & --- & --- & --- & $0,009^{*}$
\end{tabular}

${ }^{*} \mathrm{p} \leq 0,05$

Tabela 4: Resultados do Post Hoc de Tuckey para o teste de impulsão vertical (CMJ), realizado pelos atletas.

\begin{tabular}{lcccc}
\hline Variáveis & $\begin{array}{c}\text { Base } \\
\mathbf{( 3 8 , 2} \mathbf{~ c m})\end{array}$ & $\begin{array}{c}\text { Ime } \\
\mathbf{( 4 0 , 0} \mathbf{~ c m})\end{array}$ & $\begin{array}{c}\mathbf{8 h} \\
\mathbf{( 3 9 , 1} \mathbf{~ c m})\end{array}$ & $\begin{array}{c}\mathbf{2 4 h} \\
\mathbf{( 3 6 , 4} \mathbf{~ c m})\end{array}$ \\
\hline Base & ---- & 0,27 & 0,78 & 0,29 \\
IME & --- & --- & 0,79 & $0,01^{*}$ \\
$8 \mathrm{hrs}$ & ---- & --- & --- & 0,59 \\
\hline
\end{tabular}

$* \mathrm{p} \leq 0,05$

Nas tabelas 5, 6, 7, 8 e 9 encontram-se os resultados médios de pico de torque e os resultados dos testes de Post Hoc apresentados pelos atletas nos diferentes momentos de avaliação.

Com o auxílio da análise estatística, detectaram-se diferenças significativas apenas nos valores de extensão do joelho esquerdo realizada no isocinético na velocidade de $60 \%$ s e no movimento de flexão do joelho direito, executada a $180^{\circ}$ s. Nessas situações, à semelhança dos resultados de força explosiva, os valores obtidos em repouso foram os que se mostraram mais baixos em relação aos demais valores. 
Tabela 5: Valores médios de pico de torque (PT) de extensão (Ex) e flexão (FLx) das pernas direita (D) e esquerda (E), obtidos em uma velocidade de $60 \%$ s nas situações de repouso (Base), imediatamente após a realização de um trabalho aeróbio (Ime) e depois de 8 e 24 horas da execução da corrida.

\begin{tabular}{lccccc}
\hline Variáveis & Base & IME & $\mathbf{8 h}$ & 24h & “p” \\
\hline PTExD (N) & $277,9 \pm 41,9$ & $288,8 \pm 50,0$ & $288,0 \pm 49,9$ & $287,7 \pm 46,9$ & 0,64 \\
PTExE (N) & $272,5 \pm 35,1$ & $279,9 \pm 41,1$ & $297,1 \pm 53,0$ & $280,7 \pm 49,6$ & $0,03^{*}$ \\
PTFLxD (N) & $125,1 \pm 26,5$ & $160,4 \pm 25,3$ & $142,5 \pm 18,1$ & $152,4 \pm 40,1$ & 0,14 \\
PTFLxE (N) & $121,7 \pm 23,8$ & $150,0 \pm 36,6$ & $161,1 \pm 23,5$ & $147,6 \pm 36,6$ & 0,84 \\
\hline
\end{tabular}

${ }^{*} \mathrm{p} \leq 0,05$

Tabela 6: Resultados do Post Hoc de Tuckey para os valores de pico de torque de extensão de perna esquerda (PTEE).

\begin{tabular}{lcccc}
\hline Variáveis & Base (272,5) & Ime (279,9) & 8h (297,1) & 24h (280,7) \\
\hline Base & ---- & 0,75 & $0,02^{*}$ & 0,70 \\
Ime & ---- & --- & 0,14 & 0,99 \\
8hrs & ---- & ---- & --- & 0,17 \\
\hline
\end{tabular}

$* \mathrm{p} \leq 0,05$

Tabela 7 - Valores médios de pico de torque (PT) de extensão (Ex) e flexão (FLx) das pernas direita (D) e esquerda (E), obtidos em uma velocidade de $180^{\circ} / \mathrm{s}$ nas situações de repouso (Base), imediatamente após a realização de um trabalho aeróbio (Ime) e depois de 8 e 24 horas da execução da corrida.

\begin{tabular}{cccccc}
\hline Variáveis & Base & IME & $\mathbf{8 h}$ & $\mathbf{2 4 h}$ & “p” \\
\hline PTExD (N) & $165,5 \pm 22,1$ & $174,8 \pm 38,7$ & $165,8 \pm 26,2$ & $171,3 \pm 35,1$ & 0,65 \\
PTExE (N) & $164,9 \pm 29,3$ & $170,9 \pm 29,3$ & $164,8 \pm 25,2$ & $167,9 \pm 36,0$ & 0,77 \\
PTFLxD (N) & $92,0 \pm 13,0$ & $97,7 \pm 22,6$ & $117,2 \pm 35,1$ & $97,3 \pm 17,6$ & $0,02 *$ \\
PTFLxE (N) & $91,9 \pm 16,4$ & $100,9 \pm 20,2$ & $117,2 \pm 41,0$ & $100,1 \pm 22,7$ & 0,20 \\
\hline
\end{tabular}

$* \mathrm{p} \leq 0,05$

Tabela 8: Resultados do Post Hoc de Tuckey para os valores de pico de torque de flexão de perna direita (PTFD), verificados em uma velocidade de $180^{\circ} / \mathrm{s}$, nos atletas.

\begin{tabular}{lcccc}
\hline Variáveis & Base (92,0) & Ime (97,7) & $\mathbf{8 h ~ ( 1 1 7 , 2 )}$ & $\mathbf{2 4 h}(\mathbf{9 7 , 3 )}$ \\
\hline Base & --- & 0,86 & $0,02^{*}$ & 0,88 \\
Ime & ---- & --- & 0,07 & 0,99 \\
8hrs & ---- & --- & --- & 0,068 \\
\hline
\end{tabular}

${ }^{*} p \leq 0,05$

Pensar a Prática, Goiânia, v. 16, n. 2, p. 320-618, abr./jun. 2013 
Tabela 9: Valores médios de pico de torque (PT) de extensão (Ex) e flexão (FLx) da perna direita (D) e esquerda (E), obtidos em uma velocidade de 300 /s nas situações de repouso (Base), imediatamente após a realização de um trabalho aeróbio (Ime) e depois de 8 e 24 horas da execução da corrida.

\begin{tabular}{lccccc}
\hline Variáveis & Base & IME & $\mathbf{8 h}$ & $\mathbf{2 4 h}$ & “p” \\
\hline PTExD (N) & $112,4 \pm 15,4$ & $126,5 \pm 30,9$ & $119,7 \pm 18,0$ & $120,1 \pm 22,4$ & 0,36 \\
PTExE (N) & $116,0 \pm 26,8$ & $126,9 \pm 22,0$ & $121,8 \pm 17,0$ & $124,3 \pm 27,4$ & 0,41 \\
PTFLxD (N) & $73,4 \pm 13,2$ & $78,8 \pm 21,1$ & $86,0 \pm 12,5$ & $79,1 \pm 15,3$ & 0,67 \\
PTFLxE (N) & $68,8 \pm 9,1$ & $76,1 \pm 20,8$ & $77,2 \pm 2,8$ & $76,9 \pm 15,9$ & 0,55 \\
\hline
\end{tabular}

\section{Discussão}

No presente estudo, não se verificou nenhum prejuízo no desempenho da força explosiva (SJ), força explosiva elástica (CMJ) e pico de torque dos avaliados imediatamente após o trabalho aeróbio, assim como 8 e 24 horas após a corrida realizada em uma intensidade referente a $90 \%$ do limiar lático com duração de 20 minutos.

As diferenças detectadas entre os valores de força explosiva (SJ), força explosiva elástica (CMJ) e pico de torque não demonstram que o trabalho aeróbio, para esses testes, tenha provocado algum tipo de interferência, tendo em vista que os valores, que se mostraram mais baixos, foram exatamente aqueles obtidos em situações em que, teoricamente, menos deveriam ter a influência do trabalho aeróbio, ou seja, na situação de repouso e após 24 horas da corrida.

Os resultados obtidos no presente estudo vão ao encontro do modelo apresentado por Docherty e Sporer (2000) que indicam que a realização de trabalho aeróbio abaixo do limiar e trabalhos de força realizados com um número maior ou igual a 10 repetições (protocolo utilizado no isocinético), ou menor que cinco repetições (protocolo de avaliação utilizado nos saltos verticais) não se enquadram dentro da zona de interferência, onde poderia ocorrer o fenômeno da interferência (Figura 2).

Com dados semelhantes aos obtidos no presente estudo encontra-se o trabalho conduzido por Leveritt, Maclaughlin e Abernethy (2000). Neste trabalho, os autores investigaram os efeitos de uma série única de exercício aeróbio sobre o nível de força de membros inferiores de oito indivíduos com experiência em treinamento de força isocinética, isométrica e isotônica. Após oito e 32 horas dessa série de exercício aeróbio foram realizados testes a fim de medir os índices de força iso- 
cinética de extensão de joelho, isométrico e isotônico. Ao final do estudo, os autores concluíram que 50 minutos de cicloergômetro não afetaram a força isocinética, isométrica e isotônica de perna, avaliada oito e 32 horas após o exercício de resistência aeróbia.

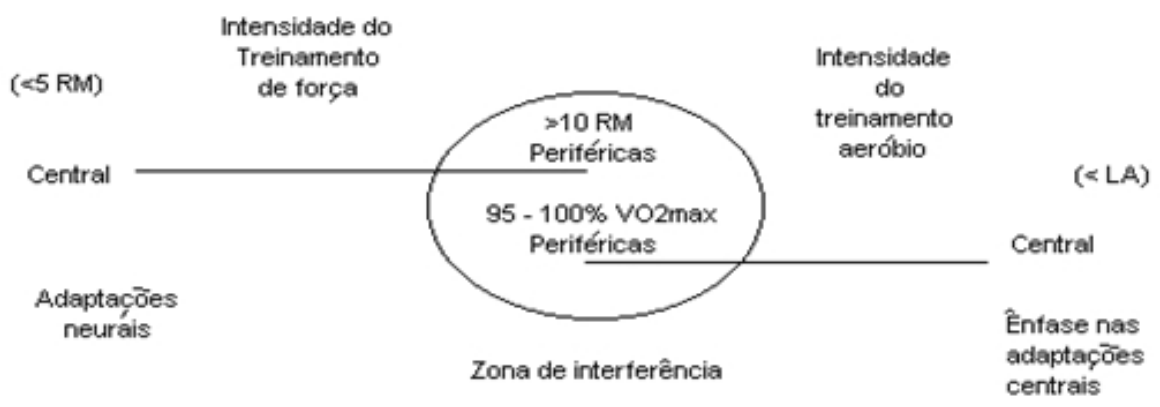

Figura 2: Modelo para o fenômeno da interferência proposto por Docherty e Sporer (2000).

Em contraposição às conclusões apresentadas no presente estudo e pelos pesquisadores Leveritt, Maclaughlin e Abernethy (2000), encontram-se os estudos realizados por Leveritt e Abernethy (1999) e Silva e Tourinho (2003).

No estudo realizado por Leveritt e Abernethy (1999), com o objetivo de determinar se o rendimento da capacidade motora força seria prejudicado após a realização de séries de exercícios aeróbios executados com uma intensidade elevada, foram analisados cinco homens e uma mulher, que realizaram exercícios de força (isotônico e isocinético) sob condição de controle (nenhuma intervenção experimental) e após as séries de exercícios aeróbios (os testes de força foram realizados após trinta minutos da execução da série de trabalho aeróbio). Por intermédio da análise dos dados, Leveritt e Abernethy (1999) puderam detectar que o exercício aeróbio executado antes das séries de força causou uma redução significativa tanto no rendimento do teste com o exercício de agachamento quanto no equipamento que mediu força isocinética em comparação à situação-controle. $\mathrm{O}$ estudo mostrou que 
a magnitude do prejuízo na força isotônica foi maior que o verificado para os exercícios isocinéticos. Para Leveritt e Abernethy (1999), parece claro que futuras pesquisas devem ser realizadas na tentativa de esclarecer os mecanismos fisiológicos da fadiga responsáveis pelo prejuízo no desempenho da força observado após a realização de séries de trabalho aeróbio. Há, também, a necessidade de se estabelecer uma duração adequada do período de recuperação entre as sessões de treinamento de força e resistência aeróbia a fim de que ambas as formas de treinamento possam ser realizadas em uma intensidade ótima.

De forma semelhante aos dados obtidos por Leveritt e Abernethy (1999), no estudo conduzido por Silva e Tourinho (2003), os pesquisadores, ao analisarem a influência de uma série única de trabalho aeróbio sobre a força muscular quando se procura integrar força e resistência aeróbia numa mesma sessão de treinamento, verificaram que trinta minutos de corrida contínua realizada em uma intensidade referente a $90 \%$ do limiar anaeróbio lático prejudicou o desempenho do teste de agachamento realizado imediatamente após o trabalho aeróbio por onze praticantes de treinamento de força.

No estudo, a média obtida no teste de agachamento realizado a $80 \%$ de 1 RM sem a realização do trabalho aeróbio precedendo ao teste foi de $14 \pm 2$ repetições. Já, quando os indivíduos realizaram a corrida contínua antes do teste, verificou-se uma diminuição significativa para $10 \pm 2$ repetições $(p \leq 0,05)$. Para Silva e Tourinho (2003), especificamente, neste este estudo, o desempenho da força muscular foi prejudicado pela série de trabalho aeróbio.

Ao confrontar as informações obtidas com relação aos efeitos do treinamento combinado sobre o ganho de força e potência aeróbia, de uma forma especulativa, torna-se possível inferir que as contradições observadas nos estudos que investigam esse assunto estejam realmente ligadas a fatores que incluem o estado inicial de treinamento dos indivíduos, o modelo de treinamento, a intensidade, o volume e a frequência do treinamento e a maneira como as duas formas de treinamento (força muscular e resistência aeróbia) foram integradas (LEVERITT et al., 1999; SALE et al., 1990a, 1990b).

Outra possível explicação que pode justificar, em parte, as controvérsias verificadas entre os estudos que investigam a combinação de treinamentos concorrentes diz respeito à seleção de variáveis dependentes. Para Leveritt et al. (1999), algumas variáveis dependentes parecem ser mais sensíveis do que outras aos efeitos do treinamento 
antagônico. Tal fato fica evidente ao se verificar as respostas dos treinamentos de força isocinético, isotônico e isométrico, quando treinados simultaneamente com resistência aeróbia. De acordo com os autores, a maioria dos estudos realizados com treinamento isotônico verificou prejuízos no desenvolvimento da força quando realizada em combinação com o treinamento aeróbio; no entanto, essa inibição no desempenho não foi verificada quando foi realizado treinamento de força isocinético em baixas velocidades de contração muscular. Desse modo, Leveritt et al. (1999) sugerem que a seleção de variáveis dependentes pode afetar a interpretação dos dados obtidos nos estudos que procuram combinar força e resistência aeróbia ao mesmo tempo.

Reforçando a hipótese levantada por Leveritt et al. (1999), de que, dependendo da variável analisada pode haver diferentes respostas ao treinamento combinado de força e resistência aeróbia, encontra-se o estudo dirigido por Lepers et al. (2000). O estudo foi elaborado para examinar os efeitos de uma corrida prolongada (duas horas a $75 \%$ da potência aeróbia de triatletas) sobre a capacidade de torque muscular dos extensores do joelho em três tipos de ações musculares: isométrica, concêntrica e excêntrica realizadas em diferentes velocidades angulares. Pela análise dos dados, os autores verificaram que, após o trabalho aeróbio executado pelos atletas, houve uma diminuição nos índices de força muscular e que essa perda na capacidade de produzir força parece ser dependente do tipo de ação muscular, já que se detectou uma maior diminuição nas ações excêntricas e isométricas em comparação às ações concêntricas. Esse comportamento ocorreu independentemente da velocidade angular em que foi realizado o movimento. Para Lepers et al. (2000), o comprometimento no desempenho da força muscular, neste estudo, parece estar mais ligado a possíveis prejuízos provocados pelo treinamento aeróbio nos mecanismos contráteis do que na atividade neural da musculatura examinada, no caso o quadríceps.

A observação sobre a existência dessa intensa controvérsia entre os estudos que analisam o fenômeno da interferência apenas vem confirmar a complexidade na análise do efeito combinado de regimes de treinamentos concorrentes sobre as respostas adaptativas do organismo frente a esse modelo de treinamento esportivo e a necessidade de mais estudos a respeito do assunto. No entanto, parece razoável sugerir que o desempenho de atividades realizadas em alta intensidade e curtíssima duração (até $10 \mathrm{seg}$ ), onde há o predomínio do processo de 
ressíntese de ATP baseado no sistema de fosfagênios (ATP/CP), não é prejudicado quando antecedido pelo trabalho aeróbio, realizado em uma intensidade abaixo do limiar anaeróbio.

\title{
Conclusões
}

O trabalho aeróbio realizado em uma intensidade referente a $90 \%$ da velocidade do limiar lático, com duração de 20 minutos, não prejudicou, no presente estudo, o desempenho da força explosiva (SJ), força explosiva elástica (CMJ), assim como o pico de torque (PT) avaliado nos movimentos de extensão e flexão dos joelhos da perna direita e esquerda de atletas, nas velocidades de 60, 180 e 300\%, imediatamente após a realização do trabalho aeróbio, bem como oito e 24 horas após a execução da corrida. Desse modo, não se verificou a presença de uma possível fadiga periférica - Hipótese aguda - que viesse a prejudicar o desempenho da força após uma série de trabalho aeróbio com estas características.

Acredita-se que novos estudos precisam ser realizados, procurando experimentar diferentes intensidades de corrida e tempo de recuperação, assim como a utilização de corridas de intensidade variada, a fim de se verificar a influência sobre o desempenho da força muscular.

\section{Acute effects of aerobic training on the muscular strength performance}

\begin{abstract}
We investigated the acute effects of an aerobic training session on the skeletal muscle strength performance. Adult athletes performed an aerobic training session followed by legs fast power and peak torque tests at rest, after the aerobic training session, after 8 and 24 hours after the aerobic training. From the results obtained, it was possible to verify that 20 minutes aerobic training at $90 \%$ of the aerobic threshold did not interfere in the muscle strength in any moments of recovery. It is possible to conclude that in this intensity and duration of aeribic training, the interference phenomenon was not observed in the studied variables.
\end{abstract}

Keywords: Muscle Strenght. Physical Education and Training. Athletic Performance.

Los efectos agudos del entrenamiento con ejercicios aeróbicos en el desempeño 


\section{de la fuerza muscular}

\section{Resumen}

Se investigaron las respuestas agudas inducidas por el entrenamiento aeróbico en el rendimiento de la fuerza muscular. Con este fin, los atletas adultos realizaron una serie de trabajos aeróbicos, y luego realizó pruebas para inferir la fuerza explosiva de las extremidades inferiores en las siguientes situaciones: en reposo, inmediatamente después del trabajo aeróbico y después de ocho y 24 horas después de la finalización del trabajo aeróbico. Basándose en estos resultados, se verificó que el trabajo aeróbico realizó durante 20 minutos a una intensidad de $90 \%$ del umbral de lactato, no afecte al rendimiento de la recuperación muscular en momentos diferentes, es decir, la intensidad y la duración de esta ejecución, no fue el fenómeno de la interferencia de las variables analizadas.

Palabras clave: Fuerza Muscular. Educación y Entrenamiento Físico. Rendimiento Deportivo.

\section{Referências}

BELL, G. et al. Effect of strength training and concurrent strength and endurance training on strength, testosterone, and cortisol. Journal of Strength and Conditioning Research, v.11, n.1, p.57-64, 1997.

BOSCO, C. et al. A dynamometer for evaluation of dynamic muscle work. European Journal of Applied Physiology, v.70, p.379-386, 1995.

DOCHERTY, D.; SPORER, B. A proposed model for examining the interference phenomenon between concurrent aerobic and strength training. Sports Medicine, v.30, n.6, p.385-394, 2000.

DOLEZAL, B. A.; POTTEIGER, J.A. Concurrent resistance and endurance training influence basal metabolic rate in nondieting individuals. Journal of Applied Physiology, v.85, n.2, p.695-700, 1998.

DUDLEY, G. A.; DJAMIL, R. Incompatibility of endurance and strength -training modes of exercise. Journal of Applied Physiology, v.59, n.5, p.1446-1451, 1985.

FELL, J. W. et al. Evaluation of the accusport lactate analyzer. International Journal of Sports Medicine, v.19, p.199-204, 1998. 
GAMBKE, B. et al. Multicenter evaluation of new portable system for determining blood lactate. Medicine and Science in Sports and Exercise, v.26, n.5, p.142, 1994.

GRAVELLE, B.T.; BLESSING, D.L. Physiological adaptation in women concurrently training for strength and endurance. Journal of Strength and Conditioning Research, v.14, n.1, p.5-13, 2000.

HECK, H. et al. Justification of the $4-\mathrm{mmol} / \mathrm{L}$ lactate threshold. International Journal of Sports Medicine, v.6, p.117-130, 1985.

HICKSON, R.C. Interference of strength development by simultaneously training for strength and endurance. European Journal of Applied Physiology, v.45, p.255-263, 1980.

KRAEMER, W. J. et al. Compatibility of high - intensity strength and endurance training on hormonal and skeletal muscle adaptations. Journal of Applied Physiology, v.78, n.3, p.976-989, 1995.

LEPERS, R. et al. The effects of a prolonged running exercise on strength characteristics. International Journal Sports Medicine, v.21, p.275-280, 2000.

LEVERITT, M.; ABERNETHY, P. J. Acute effects of high-intensity endurance exercise on subsequent resistance activity. Journal of Strength and Conditioning Research, v.13, n.1, p.47-51, 1999.

LEVERITT, M. et al. Concurrent strength and endurance training: a review. Sports Medicine, v.28, n.6, p.413-427, 1999.

LEVERITT, M.; MacLAUGHLIN, H.; ABERNETHY, P. Changes in leg strength 8 and $32 \mathrm{~h}$ after endurance exercise. Journal of Sports Sciences, v.18, p.865-871, 2000.

NELSON, A. G. et al. Consequences of combining strength and endurance training regimes. Physical Therapy, v.70, n.5, 1990.

NETER, J. et al. Applied Linear Statistical Models. 4. ed. New York, IE McGraw Hill, 1996.

OLIVEIRA, F. R.; GAGLIARDI, J. F. L.; KISS, M. A. P. D. M. Proposta de referência para a prescrição de treinamento aeróbio e anaeró- 
bio para corredores de média e longa duração. Revista Paulista de Educação Física, São Paulo, v.8, n.2, p.68-76, jul./dez., 1994

SALE, D. G. et al. Comparison of two regimens of concurrent strength and endurance training. Medicine and Science in Sports and Exercise, v.22, n.3, p.348-356, 1990a.

. Interaction between concurrent strength and endurance training. Journal of Applied Physiology, v.68, n.p.260-270, 1990 b.

SILVA, A.; TOURINHO, H. F. Resistência aeróbia e desempenho de força: o fenômeno da interferência. In: XXVI SIMPÓSIO INTERNACIONAL DE CIÊNCIAS DO ESPORTE, 2003, Anais... São Paulo. Edição Especial da Revista Brasileira de Ciência e Movimento, Brasília, p.162, 2003.

SZMUCHROWSKI, L. A.; VIDIGAL, J. M. S. Saltos no diagnóstico e prescrição das cargas de treinamento. In: SILVA, F.M. Treinamento desportivo: atualidades e perspectivas. João Pessoa: Universitária, 1999. p.97-120.

TOURINHO, H.F. Treinamento esportivo: interfaces com a fisiologia do esporte. Passo Fundo: UPF, 2007. p.89-122.

Periodização de treinamentos concorrentes e o fenômeno da interferência. Lecturas Educación Física y Deportes. v.14, p. 1-9, 2010.

Recebido em: 16/02/2012

Revisado em: 19/06/2012

Aprovado em: 22/10/2012

Endereço para correspondência

tourinho@usp.br

Hugo Tourinho Filho

Universidade de São Paulo, Escola de Educação Física e Esporte

Av. Bandeirantes, 3.900

Monte Alegre

14040-900 - Ribeirao Preto, SP - Brasil 